\title{
Evaluation of Atlas-Based Attenuation Correction for Integrated PET/MR in Human Brain: Application of a Head Atlas and Comparison to True CT-Based Attenuation Correction
}

\author{
Tetsuro Sekine ${ }^{1-3}$, Alfred Buck ${ }^{1,2}$, Gaspar Delso ${ }^{1,2,4}$, Edwin E.G.W. ter Voert ${ }^{1,2}$, Martin Huellner ${ }^{1,2,5}$, \\ Patrick Veit-Haibach ${ }^{1,2,6}$, and Geoffrey Warnock ${ }^{1,2}$ \\ ${ }^{1}$ Department of Nuclear Medicine, University Hospital Zurich, Zurich, Switzerland; ${ }^{2}$ University of Zurich, Zurich, Switzerland; \\ ${ }^{3}$ Department of Radiology, Nippon Medical School, Tokyo, Japan; ${ }^{4}$ GE Healthcare, Waukesha, Wisconsin; ${ }^{5}$ Department of \\ Neuroradiology, University Hospital Zurich, Zurich, Switzerland; and ${ }^{6}$ Division of Diagnostic and Interventional Radiology, \\ Department of Medical Radiology, University Hospital Zurich, Zurich, Switzerland
}

\begin{abstract}
Attenuation correction (AC) for integrated PET/MR imaging in the human brain is still an open problem. In this study, we evaluated a simplified atlas-based AC (Atlas-AC) by comparing ${ }^{18} \mathrm{~F}-\mathrm{FDG}$ PET data corrected using either Atlas-AC or true CT data (CT-AC). Methods: We enrolled 8 patients (median age, $63 \mathrm{y}$ ). All patients underwent clinically indicated whole-body ${ }^{18} \mathrm{~F}$-FDG PET/CT for staging, restaging, or follow-up of malignant disease. All patients volunteered for an additional PET/MR of the head (additional tracer was not injected). For each patient, 2 AC maps were generated: an Atlas-AC map registered to a patient-specific liver accelerated volume acquisition-Flex MR sequence and using a vendor-provided head atlas generated from multiple CT head images and a CT-based AC map. For comparative AC, the CT-AC map generated from PET/CT was superimposed on the Atlas-AC map. PET images were reconstructed from the list-mode raw data from the PET/MR imaging scanner using each AC map. All PET images were normalized to the SPM5 PET template, and ${ }^{18} \mathrm{~F}-\mathrm{FDG}$ accumulation was quantified in 67 volumes of interest (VOIs; automated anatomic labeling atlas). Relative difference (\%diff) between images based on Atlas-AC and CT-AC was calculated, and averaged difference images were generated. ${ }^{18} \mathrm{~F}-\mathrm{FDG}$ uptake in all VOls was compared using Bland-Altman analysis. Results: The range of error in all 536 VOls was $-3.0 \%-7.3 \%$. Whole-brain ${ }^{18} \mathrm{~F}-\mathrm{FDG}$ uptake based on Atlas-AC was slightly underestimated (\%diff $=2.19 \% \pm 1.40 \%$ ). The underestimation was most pronounced in the regions below the anterior/posterior commissure line, such as the cerebellum, temporal lobe, and central structures (\% diff $=3.69 \% \pm 1.43 \%, 3.25 \% \pm 1.42 \%$, and $3.05 \% \pm 1.18 \%$ ), suggesting that Atlas-AC tends to underestimate the attenuation values of the skull base bone. Conclusion: When compared with the gold-standard CT-AC, errors introduced using Atlas-AC did not exceed $8 \%$ in any brain region investigated. Underestimation of ${ }^{18} \mathrm{~F}-\mathrm{FDG}$ uptake was minor $(<4 \%)$ but significant in regions near the skull base.
\end{abstract}

\footnotetext{
Received Apr. 13, 2015; revision accepted Oct. 7, 2015.

For correspondence or reprints contact: Tetsuro Sekine, University Hospital Zurich, Ramistrasse 100, 8091, Zurich, Switzerland.

E-mail: tetsuro.sekine@gmail.com

Published online Oct. 22, 2015.

COPYRIGHT (c) 2016 by the Society of Nuclear Medicine and Molecular Imaging, Inc.
}

Key Words: PET/MR; attenuation correction; atlas-based; brain; 18F-FDG

J Nucl Med 2016; 57:215-220

DOI: 10.2967/jnumed.115.159228

$\sim$ ombined PET/MR systems have recently become available for clinical use $(1,2)$. Compared with CT, MR imaging provides better soft-tissue contrast, and functional imaging such as diffusion-weighted images and MR spectroscopy, yielding superior diagnostic accuracy in the brain without subjecting the patient to ionizing radiation. The combination of PET and MR imaging not only provides complementary functional information, but also has the potential to provide further advantages over PET/CT, such as prereconstruction motion correction of PET data (2,3). To obtain quantitative PET data and to be able to objectively interpret image features between patients, correction for tissue-based photon attenuation is necessary. Unlike CT, linear photon attenuation coefficients cannot be directly derived from MR imaging (4). Indeed, predicting attenuation values from MR images is difficult because MR signals are related to proton density and relaxation properties of tissues. The relative amount of bone in the head is higher than in other parts of the body, and this bone contributes significantly to the attenuation of PET photons (5). MR attenuation-correction (AC) methods for the brain should consider bone in attenuation maps to allow accurate PET quantification. As combined PET/MR has gained momentum, several AC methods have been proposed. These are roughly divided into 3 types. Atlas-based/template-based methods (6-9) generate a patient-specific AC map from a CT atlas or attenuation-map template. Segmentation methods $(10,11)$ derive the segmentation of tissue components, for example, bone, air, and brain tissue, from dedicated pulse sequences. These sequences include Dixon T1-weighted imaging (T1WI), ultrashort echo sequence, and high-resolution 3-dimensional T1WI. The third method is a direct extraction of an AC map from timeof-flight (TOF) PET emission data (12). Comprehensive reviews of current MR-based AC methods, their advantages, and limitations are available in the literature (13-15). 
For AC in the head using clinical PET/MR, AC methods should meet several requirements: robustness against intra- and interpatient variability in all brain regions; clinical AC methods should introduce minimal error compared with gold-standard CT-AC; time-consuming MR sequences should be avoided; postprocessing should not be time-consuming; and the output should be intuitive (making AC errors easy to recognize). To meet these requirements, the SIGNA PET/MR scanner (GE Healthcare) uses simplified atlasbased AC (Atlas-AC) with a single head atlas generated from multiple CT head images. However, the performance characteristics of this method have only been described for nonsimultaneous PET/ MR (16). The purpose of this study was the validation of Atlas-AC, by comparison to gold-standard CT-AC, using clinical ${ }^{18} \mathrm{~F}-\mathrm{FDG}$ data. Thus, we evaluated the accuracy of Atlas-AC and assessed to what degree AC map errors are propagated to PET images.

\section{MATERIALS AND METHODS}

This study was approved by the institutional review board. All subjects provided signed informed consent before the examinations.

\section{Patients}

We enrolled 8 patients. Statistical power analysis (R package pwr, http://www.r-project.org) indicated a required $n$ of 8.15 to detect a $5 \%$ error in ${ }^{18} \mathrm{~F}$-FDG quantification. The median patient age was $63 \mathrm{y}$ (range, 31-73 y), the average weight was $74.96 \pm 6.93 \mathrm{~kg}$ (range, 63.4$82.0 \mathrm{~kg}$ ), and the average body mass index was $26.4 \pm 3.06$ (range, 22.63-32.36). Five patients were men, and 3 were women.

\section{PET/CT and PET/MR Examination}

The PET/CT acquisition followed the standard protocol for a clinical oncology study on a clinical PET/CT scanner (Discovery 690 TOF PET/ CT; GE Healthcare). The average ${ }^{18} \mathrm{~F}$-FDG dose injected was $231.7 \pm$ 11.5 MBq (range, 210.8-244.6 MBq). First, a helical whole-body CT scan $(120 \mathrm{kV} ; 15-80 \mathrm{~mA}$ with automatic dose modulation; rotation time, $0.5 \mathrm{~s}$; helical thickness, $3.75 \mathrm{~mm}$; pitch, $39.37 \mathrm{~mm} /$ rotation; image matrix, $512 \times 512$; voxel size, $1.4 \times 1.4 \times 3.3 \mathrm{~mm}$ ) was acquired for AC of PET data (16). Then, a whole-body PET image was acquired for a period of 2 min per bed position. Immediately after the PET/CT scan, patients were moved to the integrated TOF PET/MR scanner (SIGNA PET/MR; GE Healthcare), and a brain PET/MR scan was obtained for a period of $20 \mathrm{~min}$. Patients were allowed to move freely from PET/CT to PET/MR. Once positioned for PET/MR, head movement was prevented using firm foam cushions between the head and coil. During the PET scan, liver-accelerated volume acquisition (LAVA)-Flex MR images (repetition time, $\sim 4 \mathrm{~ms}$; echo time, $2.23 \mathrm{~ms}$; flip angle, $12^{\circ}$; slice thickness, $5.2 \mathrm{~mm}$, with $2.6 \mathrm{~mm}$ overlap; 120 slices; pixel size, $1.95 \times 1.95 \mathrm{~mm}$; partial Fourier, $70.3 \%$; acquisition time, $18 \mathrm{~s}$ ) were acquired for Atlas-AC. This sequence was selected for a combination of reasons. It was used in full-body imaging for fat-water separation and thus extended to head use. Additionally, its use allowed the user to revert to 4-class segmentation AC (not considered in this study). Finally, the sequence was short, $18 \mathrm{~s}$, making it an efficient part of the PET/MR workflow. The first 3 patients underwent PET/MR with the manufacturer's standard head-and-neck coil (28-channel GEM Head and Neck Unit [GE Healthcare], as part of a whole-body imaging protocol), and the 5 subsequent patients were imaged with a standard head coil (8-channnel HD Brain; GE Healthcare).

\section{Reconstruction of AC Map}

For each patient, 2 AC maps were generated, Atlas-AC and CT-AC. The Atlas-AC map was registered to the LAVA-Flex in-phase image using a dedicated process developed by the manufacturer (Fig. 1). An atlas-based method was used to derive a pseudo-CT that included continuous attenuation information for the head, using a single-head atlas. This atlas was provided by the vendor and is based on CT images from 50 subjects (16). The patient-specific pseudo-CT was generated on the PET/MR scanner as follows. First, 3-mm Hessian-bone enhancement from LAVA in-phase images was performed. Second, application of rigid and nonrigid B-spine-based elastic registration between boneenhanced MR image and the head atlas was performed (16). Third, the head contour and segmentation of tissue and sinus was generated. Fourth, the registered head atlas from step 2 and the head contour from step 3 were combined to generate pseudo-CT. Fifth, the AC map was generated from the pseudo-CT using the standard vendor-provided CT lookup tables. Finally, the MR coil was added to the AC map. This entire procedure took less than $30 \mathrm{~s}$, without user interaction. A more detailed description of the algorithm is provided in Wollenweber et al. (16). The second AC map was the coregistered CT-AC map, generated as follows. First, the original CT-AC map was exported from the PET/CT scanner. Second, from this map, the CT bed was removed manually. Third, the threshold was set to extract the outside air component from the CT-AC map. None of the images used in this study contained large artifacts likely to affect air thresholding. Large dental artifacts in future patients would be transferred to the images as part of the Atlas-AC algorithm, making additional quality control of the resultant AC map necessary. Fourth, we performed manual linear registration between the $2 \mathrm{AC}$ maps. Finally, the map was superimposed on and coregistered to the Atlas-AC map, replacing it, manually. The second, third, and fourth steps were performed using custom Matlab (7.11.0; The MathWorks) routines.

\section{Reconstruction of PET Images}

Only the PET data from the PET/MR examination were used, reconstructed with AC based on either Atlas-AC or CT-AC maps. All PET images were reconstructed from the list-mode raw data on the PET/MR scanner. The PET reconstruction parameters were fully 3-dimensional ordered-subsets expectation maximization iterative reconstruction (17); TOF on; point-spread function modeling on; filter cutoff, $3 \mathrm{~mm}$; axial filter medium; subsets, 28; iterations, 8; scatter correction on; and pixel size, $1.17 \times 1.17 \mathrm{~mm}(18)$.

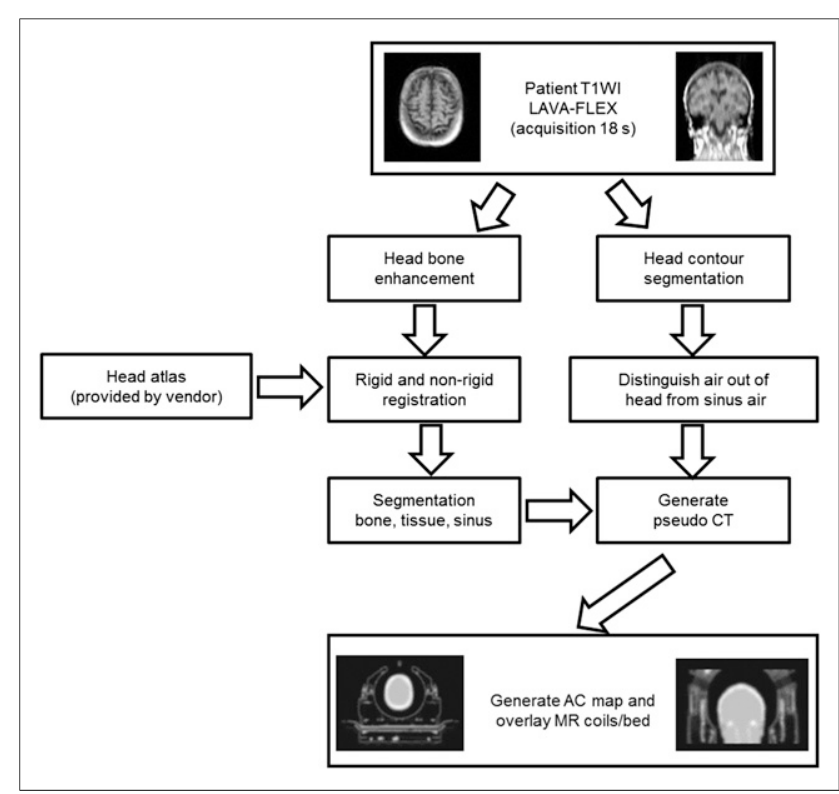

FIGURE 1. Overview of Atlas-based pseudo-CT and AC map processing. All computational time less than $30 \mathrm{~s}$. More detailed description is provided in Wollenweber et al. (16). 


\section{Analysis}

All PET images were spatially normalized to a brain template (SPM5; University College London), and 67 volumes of interest (VOIs) (automated anatomic labeling [AAL] atlas) were applied (19). Each matching AC map was also normalized using the transformation generated during the normalization of the PET images.

\section{Visual Analysis}

Visual assessment was performed on 2 levels. A radiologist with $10 \mathrm{y}$ of experience in brain imaging confirmed the absence of major artifacts in LAVA-Flex images likely to negatively affect registration. After generation of Atlas-AC and linear coregistration between Atlas$\mathrm{AC}$ and CT-AC, the fused images were examined to confirm that the resulting atlas $\mathrm{AC}$ map was also artifact-free.

Relative difference (\%diff) maps of PET and AC map images for each patient (Atlas-AC minus CT-AC, divided by CT-AC) were generated. Averages of the \%diff maps from all patients were subsequently generated.

\section{Quantitative Analysis}

Evaluation was performed in 4 steps. Whole-brain ${ }^{18} \mathrm{~F}$-FDG uptake was compared using a paired $t$ test with a significance level of 0.05 . ${ }^{18} \mathrm{~F}$-FDG uptake in 67 AAL VOIs in each of the 8 patients was compared using Bland-Altman analysis. VOIs from the AAL atlas were merged into more generalized VOIs: central structures (caudate nucleus, putamen, pallidum, thalamus), cerebellum, frontal lobe, temporal lobe, insula and cingulate gyrus, occipital lobe, parietal lobe (Supplemental Fig. 1; supplemental materials are available at http://jnm.snmjournals. org), right and left hemisphere, and cortical and subcortical regions in each hemisphere. The \%diff was calculated in each merged region and compared using a paired $t$ test with Bonferroni adjustment. Finally, after calculation of \%diff in all 67 VOIs, the VOIs were merged into 6 groups with similar \%diff pattern using group-average agglomerative clustering (SPSS Statistics 19.0.0; IBM). \%diff in each merged region was compared using a paired $t$ test with Bonferroni adjustment.

Visual and quantitative analyses were performed using PMOD 3.6 (PMOD Technologies Ltd.)

\section{RESULTS}

All 8 patients successfully underwent PET/CT and PET/MR examinations. The mean time between ${ }^{18} \mathrm{~F}$-FDG injection and the PET/MR scan was $106.8 \pm 8.3 \mathrm{~min}$ (range, 91.0-120.0 min). One patient had a small posttraumatic brain injury with lack of ${ }^{18} \mathrm{~F}-\mathrm{FDG}$ uptake. The others had no pathologic findings in the brain.

\section{Visual Analysis}

The upper cranial part of the Atlas-AC map appeared to have an attenuation value similar to that of the CT-AC map (Fig. 2; first, second, and third rows). However, the attenuation coefficients were underestimated in the Atlas-AC map for the mastoid process region, temporal bone, and skull base compared with CT-AC. This skull-underestimation caused an underestimation of ${ }^{18} \mathrm{~F}-\mathrm{FDG}$ uptake in the regions close to the skull base (Fig. 2; bottom row). Detailed examination of the CT-AC maps also showed that the attenuation coefficient of skull was not homogeneous. The AC value of temporal bone was about $0.11 \mathrm{~cm}^{-1}$ whereas the superior skull bone had a higher attenuation value, about $0.13 \mathrm{~cm}^{-1}$. In contrast, Atlas-AC applies a lower value to temporal bone $\left(\sim 0.10 \mathrm{~cm}^{-1}\right)$ and a similar value to superior bone $\left(0.13 \mathrm{~cm}^{-1}\right)$. Additional difference images are illustrated in Supplemental Figure 2.

\section{Quantitative Analysis}

Whole-brain ${ }^{18} \mathrm{~F}$-FDG uptake based on Atlas-AC was slightly but significantly underestimated $(\% \operatorname{diff}=2.19 \% \pm 1.40 \%, P<0.01)$

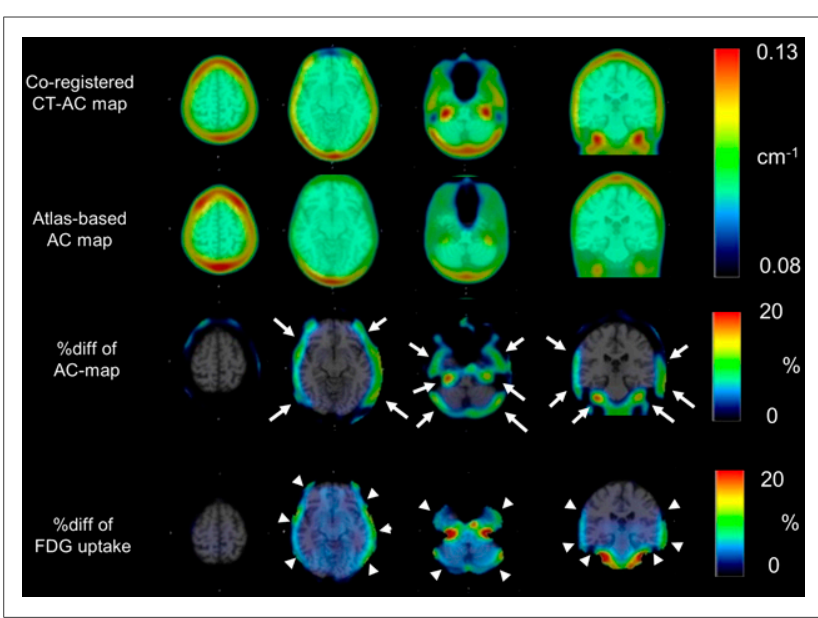

FIGURE 2. (First row) Coregistered CT-AC map. (Second row) AtlasAC map. (Third row) \%diff of AC map. (Fourth row) \%diff of ${ }^{18} \mathrm{~F}-\mathrm{FDG}$ uptake. All images were averaged from 8 patient-specific images normalized to template. Values outside CT-AC map were masked. Further information is provided in Supplemental Figure 2.

(Table 1). Linear regression showed that the ${ }^{18} \mathrm{~F}$-FDG uptake values from each AC method were highly correlated $\left(R^{2}>0.99\right)$ to a straight line with a slope of 0.966 (Fig. 3A). The Bland-Altman plot for all VOIs highlighted the trend for Atlas-AC to underestimate CT-AC (upper limit, 6.19\%; lower limit, $-1.71 \%$; range, $-3.0 \%$ to $\sim 7.3 \%$ ) (Fig. $3 \mathrm{~B}$ ) and that this bias was consistent. The underestimation was most pronounced in the regions near the skull base and below the anterior-posterior commissure line, for example, the cerebellum (\%diff $=3.69 \% \pm 1.43 \%, P<0.01)$,

TABLE 1

Relative Difference in ${ }^{18} \mathrm{~F}-\mathrm{FDG}$ Uptake $(\mathrm{kBq} / \mathrm{mL})$ Between CT-AC and Atlas-AC in Each VOI

\begin{tabular}{lll}
\hline \multicolumn{1}{c}{ Region } & \%diff & SD \\
\hline Cerebellum & $3.69 \%^{*}$ & $1.43 \%$ \\
\hline Temporal lobe & $3.25 \%^{\dagger}$ & $1.42 \%$ \\
\hline Central structure & $3.05 \%^{\dagger}$ & $1.18 \%$ \\
\hline Frontal lobe & $1.59 \%$ & $1.23 \%$ \\
\hline Insula and cingulate gyri & $1.60 \%$ & $1.27 \%$ \\
\hline Occipital lobe & $2.12 \%$ & $2.08 \%$ \\
\hline Parietal lobe & $1.63 \%$ & $1.70 \%$ \\
\hline Total & $2.19 \%^{*}$ & $1.40 \%$ \\
\hline Hemisphere left & $2.28 \%{ }^{\ddagger}$ & $1.41 \%$ \\
\hline Hemisphere right & $2.09 \%^{\ddagger}$ & $1.42 \%$ \\
\hline Cortical left & $2.07 \%$ & $1.44 \%$ \\
\hline Cortical right & $1.88 \%$ & $1.47 \%$ \\
\hline Subcortical left & $2.92 \%{ }^{\ddagger}$ & $1.17 \%$ \\
\hline Subcortical right & $2.86 \%{ }^{\ddagger}$ & $1.13 \%$ \\
\hline${ }^{*} P<0.01$. & & \\
${ }^{\dagger} P<0.05$. & & \\
${ }^{\ddagger} P<0.05$. & & \\
$\%$ diff $=$ CT-AC minus Atlas-AC, divided by CT-AC. & \\
\hline
\end{tabular}




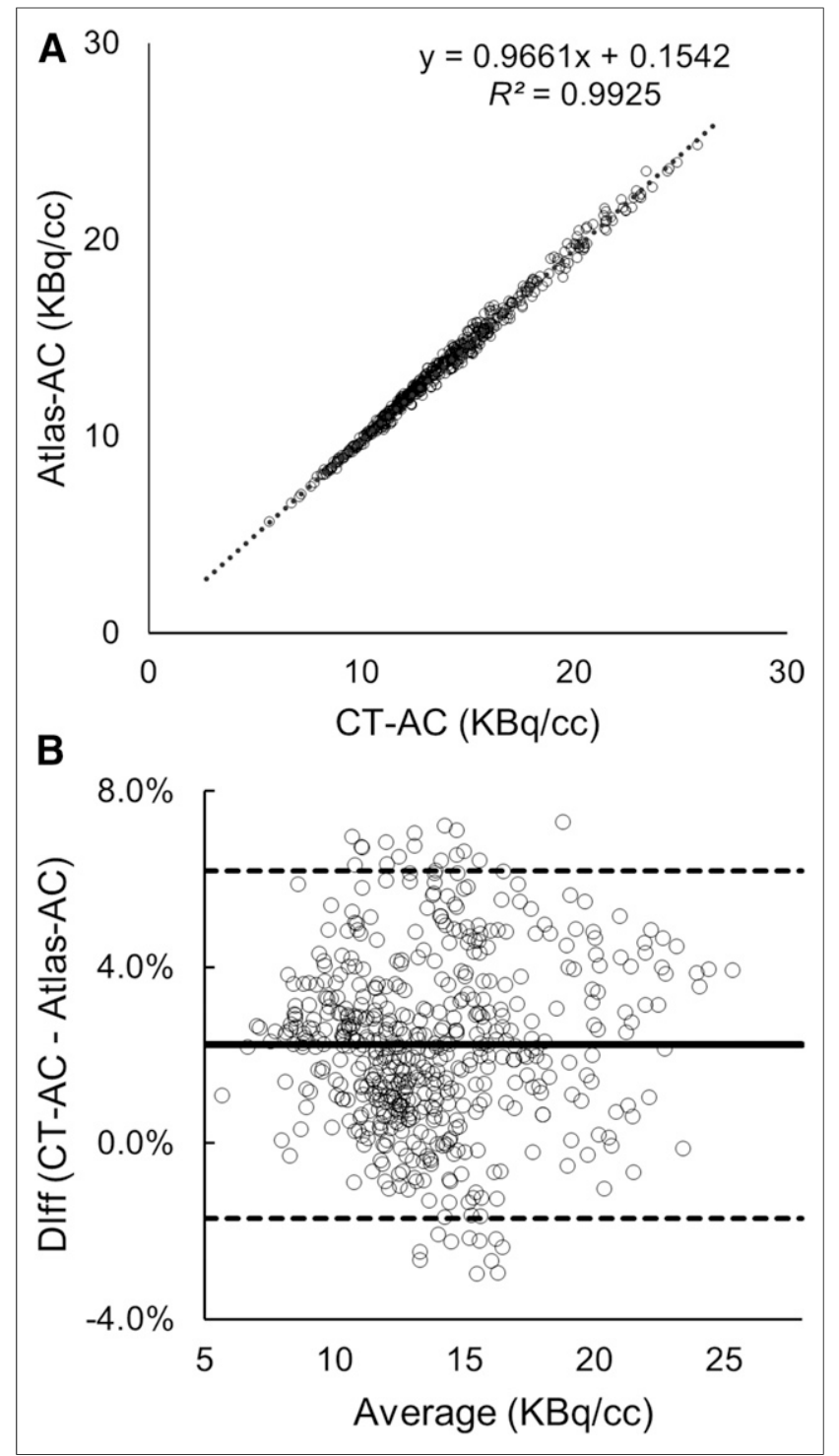

FIGURE 3. Regression (A) and Bland-Altman plots (B) for 67 VOls in 8 patients.

temporal lobe $(3.25 \% \pm 1.42 \%, P<0.05)$, central structures $(3.05 \% \pm 1.18 \%, P<0.05)$, and subcortical region (left, $2.92 \% \pm$ $1.71 \%, P<0.05$; right, $2.86 \% \pm 1.13 \%, P<0.05$ ) (Table 1). Post hoc statistical power analysis using the data from the cerebellum indicated that the expected power (0.8) was exceeded (0.865). The underestimation was more clearly depicted in the merged regions after group-average agglomerative clustering analysis (Supplemental Fig. 3). The underestimation was significant in 2 of 6 groups and pronounced in group $1(2.80 \% \pm 1.27 \%, P<0.05)$ and group 2 $(3.79 \% \pm 1.50 \%, P<0.01)$ (Table 2$)$. Groups $3-6$ showed only nonsignificant underestimation. Groups 1 and 2 cover most regions near the skull base. Visualization of the grouped regions from the clustering analysis highlighted that the Atlas-AC tended to underestimate ${ }^{18}$ F-FDG uptake near the skull base (Fig. 4).

\section{DISCUSSION}

In this study, we compared a simplified atlas-based AC method with the CT-AC gold standard using ${ }^{18}$ F-FDG PET data from pa- tients imaged in a recently developed integrated PET/MR scanner (SIGNA PET/MR). This study revealed 3 major findings. First, the Atlas-AC method was comparable to CT-AC. The range of error in all 536 VOIs analyzed was less than $8 \%(-3.0 \%-7.3 \%)$. Second, the attenuation coefficients for temporal and skull base bone tended to be slightly underestimated by atlas-based AC. Third, this error resulted in minor underestimation of ${ }^{18} \mathrm{~F}-\mathrm{FDG}$ uptake near the skull base.

To our knowledge, this is the first study to evaluate the performance of head AC on GE Healthcare's integrated PET/MR scanner (16). There have been few AC-comparison studies using a combination of PET/CT and PET/MR data (5,20-23). In clinical PET/MR, patient motion or geometric distortions of MR images can create a mismatch between PET and MR data. Simulation studies using PET/CT and an independent MR scanner may not fully account for these effects. For that reason, it is advantageous to work directly on an integrated PET/MR scanner, reflecting the desired clinical situation.

Correcting for attenuation due to skull bone is critical in brain PET studies because the head is compact, and the cortical bone is a high-attenuation tissue for PET photons. Completely neglecting bone tissue attenuation leads to large underestimations $(>20 \%)$ of the radiotracer concentration in the cortex (24). Lines of response (LORs) at a tangent to the surface of the brain, traversing the skull at an oblique angle, have a longer path through skull bone than central LORs perpendicular to the surface of the brain. Therefore, total attenuation along these LORs is higher than that of central LORs. Consequently, the overall effect of AC error is larger at the edge than at the center, leading to a regionally variable bias (5). Such a bias is evident in Figure 2.

Calculation of the percentage difference between Atlas-AC and CT-AC maps illustrated an underestimation of AC values in the temporal and skull base bone regions, explaining the resultant underestimation of ${ }^{18} \mathrm{~F}-\mathrm{FDG}$ uptake in temporal and cerebellar regions. This is likely caused by errors in the segmentation of enhanced bone from in-phase T1WI and the registration of the head atlas during Atlas-AC map calculation. Jaw movement and differences in neck position between scans may also introduce errors. In regions near the base of the skull, the bone is more axially aligned, so that a small error in the AC map leads to high

TABLE 2

Relative Difference in ${ }^{18} \mathrm{~F}-\mathrm{FDG}$ Uptake $(\mathrm{kBq} / \mathrm{mL})$ Between CT-AC and Atlas-AC in Merged VOI After Group-Average Agglomerative Clustering Analysis

\begin{tabular}{lcc}
\hline Region & \%diff & SD \\
\hline Group 1 & $2.80 \%^{\star}$ & $1.27 \%$ \\
\hline Group 2 & $3.79 \%^{\dagger}$ & $1.50 \%$ \\
Group 3 & $2.61 \%$ & $2.04 \%$ \\
Group 4 & $1.52 \%$ & $1.81 \%$ \\
Group 5 & $0.70 \%$ & $1.43 \%$ \\
Group 6 & $1.23 \%$ & $1.58 \%$ \\
Total & $2.19 \%^{\dagger}$ & $1.40 \%$ \\
\hline & & \\
${ }^{*} P<0.05$. & & \\
${ }^{\dagger} P<0.01 ;$ & & \\
${ }^{*}$ diff $=$ CT-AC minus Atlas-AC, divided by CT-AC. & \\
\hline
\end{tabular}


attenuation bias. The addition of line sources to PET/MR scanners would aid comparison in truly simultaneous acquisition.

In other recent studies of AC approaches for PET/MR, the mean absolute relative percentage errors between PET images were reported as $2.42 \% \pm 1.0(20), 2.55 \% \pm 0.86(21), 1.38 \% \pm 4.52$ (23), and $1.82 \% \pm 2.45(22)$. Care should be taken when comparing these studies and the present study, because of methodologic variations. However, the mean error across all 536 VOIs in this study, $2.24 \% \pm 1.98$, is generally comparable. In studies using the AAL atlas for regional VOI analysis of ${ }^{18} \mathrm{~F}-\mathrm{FDG}$ accumulation, the mean error and SD in the cerebellum were larger than in other regions $(21,22)$. This result is consistent with ours.

Although all quantitation errors were less than $8 \%$, the biased distribution of the errors means that care is required when assessing subtle changes in PET tracer uptake, such as reductions in temporal lobe ${ }^{18} \mathrm{~F}$-FDG uptake in Alzheimer disease, or when comparing studies acquired on both PET/CT and PET/MR scanners. Fortunately, clinically significant reductions in ${ }^{18} \mathrm{~F}-\mathrm{FDG}$ uptake tend to be larger than the errors found due to Atlas-AC (e.g., 14\%-24\% (25)) and often involve other cortical regions. Particular care should be taken when comparing kinetic modeling studies from PET/MR with PET/ $\mathrm{CT}$, in which the cerebellum is commonly used as a reference tissue. Additionally, age, sex, and racial differences in skull thickness/density should be considered if a single atlas is used (26).

Point measurements in the CT-AC map demonstrate that head bone attenuation values vary by region, likely due in part to partialvolume effects. The AC value of temporal bone (measured in the average CT-AC map) was about $0.11 \mathrm{~cm}^{-1}$, whereas the upper cranium had a higher value, about $0.13 \mathrm{~cm}^{-1}$, suggesting that simple segmentation of head bone and assignment of a fixed AC value to cortical regions is less accurate than using a bone atlas with continuous values (27). Because temporal bone is thinner than that in the upper cranium, partial-volume effects may be more pronounced.

Wollenweber et al. (16) compared a similar atlas-based AC method with PET/CT with CT-AC using an independent MR scanner (with dedicated cross-modality patient shuttle) in 13 patients. They reported no statistically significant under- or overestimation of ${ }^{18} \mathrm{~F}-\mathrm{FDG}$ uptake when replacing $\mathrm{CT}$-AC with atlas-based $\mathrm{AC}$, and overall vari-

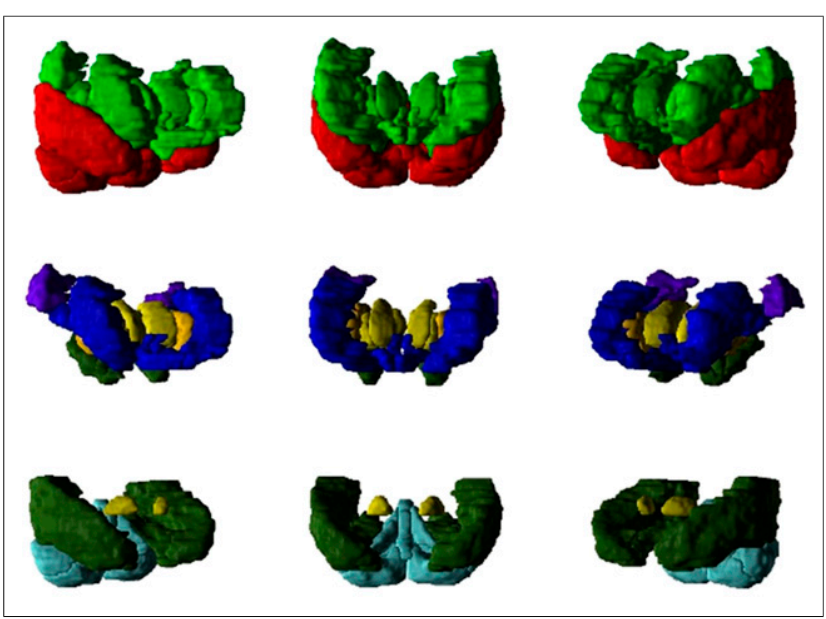

FIGURE 4. Merged skull base regions after group-average agglomerative clustering. Green = group 1; red = group 2 . (Second row) VOls in group 1. (Third row) VOls in group 2. Blue = frontal; green = temporal; red $=$ occipital; purple $=$ parietal; brown = insula/cingulate; yellow $=$ central structures; light blue = cerebellum (Supplemental Fig. 1). ability was small. This contrast to our findings likely occurred because they defined only VOIs in the cerebrum. Using the complete AAL VOI set in this study ensured that cerebellum, the most underestimated region, was included.

In this study, cluster analysis indicated that cerebrum regions could be grouped, that is, resulting groups 3-6, and that the statistical error in these groups was nonsignificant. This is consistent with the results of Wollenweber et al. Cluster analysis was used to combine regions with similar \%diff patterns. The resulting clusters did not correspond directly to discrete anatomic-functional brain regions but confirmed that underestimated ${ }^{18} \mathrm{~F}$-FDG uptake is generally confined to the skull base area. Additionally, cluster analysis confirmed that there was no asymmetry between left and right hemispheres.

Segmentation of MR images from a dedicated sequence such as ultrashort echo time represents another solution for AC in PET/MR studies. However, using pure parametric maps for AC may lead to bias in regions neighboring bone and air and in areas of high magnetic field inhomogeneity (28). Advantages of atlas-based methods include the ability to assign continuous AC values, robustness, and reduced sensitivity to acquisition artifacts. However, atlas-based methods are not generally suitable for the evaluation of postoperative patients or patients with implants. To address these problems, a combination of dedicated segmentation methods with atlas-based methods $(20,23,29)$, with neural networks $(29,30)$ or with estimation of posterior probability $(22,31)$, may be required. The drawbacks of these state-of-art methods are additional acquisitions, discrete map preparation, and prolonged computational time. Thus, the gold-standard method in AC for PET/MR has yet to be determined. In the current clinical setting, it is important to evaluate PET images based on AC derived from MR images with a good understanding of the differences to CT-AC.

A limitation of our study is that the number of patients included is small. However, using automated methods where possible, we aimed to reduce variation. The statistical power was sufficient to detect underestimation of ${ }^{18} \mathrm{~F}$-FDG uptake in discrete regions.

Artifacts from dental implants may cause substantial errors in AC maps based in any way on MR images. However, in this study, no patients had severe dental artifacts, so we were unable to assess their impact.

\section{CONCLUSION}

The simplified atlas-based AC method provided for brain imaging on the SIGNA PET/MR scanner proved comparable to the goldstandard CT-AC. However, there was a slight underestimation of PET tracer uptake values, especially near the base of the skull.

\section{DISCLOSURE}

The costs of publication of this article were defrayed in part by the payment of page charges. Therefore, and solely to indicate this fact, this article is hereby marked "advertisement" in accordance with 18 USC section 1734. This study was funded by IIS grants from Bayer Healthcare, Roche Pharmaceutical, GE Healthcare, and Siemens Medical Solutions. Patrick Veit-Haibach received speaker fees from GE Healthcare. Gaspar Delso is an employee of GE Healthcare. Only non-GE employees had control of inclusion of data and information that might present a conflict of interest for authors who are employees of GE Healthcare. Patients were acquired as part of a GE Healthcare-sponsored CE Certification study. No other potential conflict of interest relevant to this article was reported. 


\section{REFERENCES}

1. Iagaru A, Mittra E, Minamimoto R, et al. Simultaneous whole-body time-offlight ${ }^{18}$ F-FDG PET/MRI: a pilot study comparing SUVmax with PET/CT and assessment of MR image quality. Clin Nucl Med. 2015;40:1-8.

2. Nensa F, Beiderwellen K, Heusch P, Wetter A. Clinical applications of PET/MRI: current status and future perspectives. Diagn Interv Radiol. 2014;20:438-447.

3. Werner P, Barthel H, Drzezga A, Sabri O. Current status and future role of brain PET/MRI in clinical and research settings. Eur J Nucl Med Mol Imaging. 2015;42: 512-526.

4. Carney JP, Townsend DW, Rappoport V, Bendriem B. Method for transforming CT images for attenuation correction in PET/CT imaging. Med Phys. 2006;33: 976-983.

5. Andersen FL, Ladefoged CN, Beyer T, et al. Combined PET/MR imaging in neurology: MR-based attenuation correction implies a strong spatial bias when ignoring bone. Neuroimage. 2014;84:206-216.

6. Hofmann M, Steinke F, Scheel V, et al. MRI-based attenuation correction for PET/MRI: a novel approach combining pattern recognition and atlas registration. J Nucl Med. 2008;49:1875-1883.

7. Schreibmann E, Nye JA, Schuster DM, Martin DR, Votaw J, Fox T. MR-based attenuation correction for hybrid PET-MR brain imaging systems using deformable image registration. Med Phys. 2010;37:2101-2109.

8. Malone IB, Ansorge RE, Williams GB, Nestor PJ, Carpenter TA, Fryer TD. Attenuation correction methods suitable for brain imaging with a PET/MRI scanner: a comparison of tissue atlas and template attenuation map approaches. J Nucl Med. 2011;52:1142-1149.

9. Burgos N, Cardoso MJ, Thielemans K, et al. Attenuation correction synthesis for hybrid PET-MR scanners: application to brain studies. IEEE Trans Med Imaging. 2014;33:2332-2341.

10. Berker Y, Franke J, Salomon A, et al. MRI-based attenuation correction for hybrid PET/MRI systems: a 4-class tissue segmentation technique using a combined ultrashort-echo-time/Dixon MRI sequence. J Nucl Med. 2012;53:796-804.

11. Zaidi H, Montandon ML, Slosman DO. Magnetic resonance imaging-guided attenuation and scatter corrections in three-dimensional brain positron emission tomography. Med Phys. 2003;30:937-948.

12. Rezaei A, Defrise M, Nuyts J. ML-reconstruction for TOF-PET with simultaneous estimation of the attenuation factors. IEEE Trans Med Imaging. 2014;33: $1563-1572$.

13. Wagenknecht G, Kaiser HJ, Mottaghy FM, Herzog H. MRI for attenuation correction in PET: methods and challenges. MAGMA. 2013;26:99-113.

14. Keereman V, Mollet P, Berker Y, Schulz V, Vandenberghe S. Challenges and current methods for attenuation correction in PET/MR. MAGMA. 2013;26:81-98.

15. Bezrukov I, Mantlik F, Schmidt H, Scholkopf B, Pichler BJ. MR-based PET attenuation correction for PET/MR imaging. Semin Nucl Med. 2013;43:45-59.

16. Wollenweber SD, Ambwani S, Delso G, et al. Evaluation of an Atlas-based PET head attenuation correction using PET/CT \& MR patient data. IEEE Trans Nucl Sci. 2013;60:3383-3390.
17. Hudson HM, Larkin RS. Accelerated image reconstruction using ordered subsets of projection data. IEEE Trans Med Imaging. 1994;13:601-609.

18. Bettinardi V, Presotto L, Rapisarda E, Picchio M, Gianolli L, Gilardi MC. Physical performance of the new hybrid PETCT Discovery-690. Med Phys. 2011;38:5394-5411.

19. Tzourio-Mazoyer N, Landeau B, Papathanassiou D, et al. Automated anatomical labeling of activations in SPM using a macroscopic anatomical parcellation of the MNI MRI single-subject brain. Neuroimage. 2002;15:273-289.

20. Chen Y, Juttukonda M, Su Y, et al. Probabilistic air segmentation and sparse regression estimated pseudo CT for PET/MR attenuation correction. Radiology. 2015;275:562-569.

21. Juttukonda MR, Mersereau BG, Chen Y, et al. MR-based attenuation correction for PET/MRI neurological studies with continuous-valued attenuation coefficients for bone through a conversion from R2* to CT-Hounsfield units. Neuroimage. 2015;112:160-168.

22. Poynton CB, Chen KT, Chonde DB, et al. Probabilistic atlas-based segmentation of combined T1-weighted and DUTE MRI for calculation of head attenuation maps in integrated PET/MRI scanners. Am J Nucl Med Mol Imaging. 2014;4:160-171.

23. Izquierdo-Garcia D, Hansen AE, Forster S, et al. An SPM8-based approach for attenuation correction combining segmentation and nonrigid template formation: application to simultaneous PET/MR brain imaging. J Nucl Med. 2014;55: 1825-1830.

24. Catana C, van der Kouwe A, Benner T, et al. Toward implementing an MRIbased PET attenuation-correction method for neurologic studies on the MR-PET brain prototype. J Nucl Med. 2010;51:1431-1438.

25. Klunk WE, Engler H, Nordberg A, et al. Imaging brain amyloid in Alzheimer's disease with Pittsburgh Compound-B. Ann Neurol. 2004;55:306-319.

26. Adeloye A, Kattan KR, Silverman FN. Thickness of the normal skull in the American Blacks and Whites. Am J Phys Anthropol. 1975;43:23-30.

27. Navalpakkam BK, Braun H, Kuwert T, Quick HH. Magnetic resonance-based attenuation correction for PET/MR hybrid imaging using continuous valued attenuation maps. Invest Radiol. 2013;48:323-332.

28. Delso G, Carl M, Wiesinger F, et al. Anatomic evaluation of 3-dimensional ultrashort-echo-time bone maps for PET/MR attenuation correction. $\mathrm{J}$ Nucl Med. 2014;55:780-785.

29. Santos Ribeiro A, Rota Kops E, Herzog H, Almeida P. Hybrid approach for attenuation correction in PET/MR scanners. Nucl Instrum Methods Phys Res A. 2014;734:166-170.

30. Santos Ribeiro A, Rota Kops E, Herzog H, Almeida P. Skull segmentation of UTE MR images by probabilistic neural network for attenuation correction in PET/MR. Nucl Instrum Methods Phys Res A. 2013;702:114-116.

31. Delso G, Zeimpekis K, Carl M, Wiesinger F, Hüllner M, Veit-Haibach P. Clusterbased segmentation of dual-echo ultra-short echo time images for PET/MR bone localization. EJNMMI Phys. 2014;1:7-19. 